\title{
Tuberculosis and Tumor Lysis Syndrome-Coincidence or Coexistent: A Case Report
}

\author{
Viresh Swami ${ }^{1}$, AV Lalitha², Anjan Kumar $\mathrm{TK}^{3}$
}

\begin{abstract}
Extrapulmonary tuberculosis (TB) involving bone marrow can present with various manifestations, including pancytopenia, maturation arrest, hemophagocytic lymphohistiocytosis $(\mathrm{HLH})$, or infiltration of the bone marrow by caseating or noncaseating granulomas causing reversible or irreversible fibrosis. Tumor lysis syndrome (TLS) is an oncologic emergency resulting from massive tumor cell lysis. Children with TB with bone marrow involvement may also present with laboratory features of TLS resulting from high catabolism and concomitant acute kidney injury (AKI), making the diagnosis difficult at times. We present a case of disseminated TB who presented to emergency with pancytopenia, AKI, and laboratory features of TLS.

Keywords: Bone marrow tuberculosis, Pediatric extrapulmonary tuberculosis, Severe sepsis with hyperuricemia, Tuberculosis with pancytopenia, Tumor lysis syndrome.

Indian Journal of Critical Care Medicine (2020): 10.5005/jp-journals-10071-23359
\end{abstract}

\section{INTRODUCTION}

Tuberculosis is still a burden in the developing world. Caused by Mycobacterium tuberculosis, it presents with various organ involvements along with lungs. Extrapulmonary tuberculosis (TB) can be easily missed unless a high index of suspicion. Hematological abnormalities in the form of pancytopenia, anemia, and neutropenia are seen with bone marrow involvement. ${ }^{1}$

Tumor lysis syndrome (TLS) is defined as a combination of metabolic and electrolyte abnormalities that occur in patients with cancer, usually after the initiation of cytotoxic treatment and spontaneously. It is characterized by excessive cell lysis resulting in hyperuricemia, hyperphosphatemia, hyperkalemia, and hypocalcemia. $^{2-4}$

We present a case of TB with bone marrow involvement presenting as pancytopenia with hepatosplenomegaly and laboratory findings of TLS, challenging the diagnosis.

\section{Case Description}

A 2-year-old female child presented with a history of fever for 10 days, lethargy, and breathing difficulty for 3-4 days with blue-black patches over the skin for the last 3 days. At presentation to the emergency, the child was sick with increased work of breathing, pale, and irritable. The airway was maintainable with tachypnea and subcostal and intercostal retractions and was maintaining saturation of $90 \%$ in room air. The child had a heart rate of 164/ minute, feeble peripheral pulses, and blood pressure of 90/70. The child had petechiae and ecchymotic rashes, and a throat examination revealed exudative tonsillitis. Anthropometry was done after stabilization showed severe wasting and stunting (weight for height below $-3 z$ score) suggestive of severe acute malnutrition. A respiratory examination showed bilateral crepitations with scattered rhonchi, and an abdomen examination revealed hepatosplenomegaly.

The child had a background history of recurrent episodes of fever for 3 months, with progressive lethargy and decreased activity since 1 week. She also had bloody purulent ear discharge from both
${ }^{1-3}$ Department of Pediatric Critical Care, St. John's Medical College, Bengaluru, Karnataka, India

Corresponding Author: AV Lalitha, Department of Pediatric Critical Care, St. John's Medical College, Bengaluru, Karnataka, India, Phone: +91 9448461673, e-mail: drlalitha03@gmail.com

How to cite this article: Swami V, Lalitha AV, TK Anjan kumar. Tuberculosis and Tumor Lysis Syndrome-Coincidence or Coexistent: A Case Report. Indian J Crit Care Med 2020;24(2):145-147.

Source of support: Nil

Conflict of interest: None

ears since 1 month. She was treated as inpatient admission with the course of antibiotics and blood transfusion with documented pancytopenia 1 week back.

In the emergency room, the child was started on oxygen with nonrebreathing mask; received fluid resuscitation, vasopressors, and the first dose of antibiotics; and shifted to the pediatric intensive care unit. The child received respiratory support in the form of noninvasive ventilation (NIV). The initial laboratory parameters were hemoglobin $3.6 \mathrm{~g} / \mathrm{dL}$, total leucocyte count (TLC) $1,810 / \mathrm{mm}^{3}$, absolute neutrophil count (ANC) $254 / \mathrm{mm}^{3}$, platelet $2,000 / \mathrm{mm}^{3}$, and lactate $9.2 \mathrm{mmol} / \mathrm{L}$.

A provisional diagnosis of Leukemia with febrile neutropenia was made. Further workup showed high uric acid ( $16.6 \mathrm{mg} / \mathrm{dL})$, high phosphorus ( $7.0 \mathrm{mg} / \mathrm{dL})$, and hyperkalemia $(5.8 \mathrm{mEq} / \mathrm{L})$ suggestive of TLS. The child also had stage I acute kidney injury (AKI). The child was given one dose of injection rasburicase along with volume resuscitation to maintain adequate urine output and was continued on allopurinol for 48 hours till uric acid levels normalized. Blood culture on admission revealed pseudomonas growth. She was continued on injection ceftazidime, and amikacin (renal corrected dose) was added.

Peripheral smear showed microcytic, hypochromic red blood cells, with few blast cells and thrombocytopenia. Bone marrow aspiration and biopsy showed hypercellular marrow with normoblastic, erythroid maturation with $2 \%$ blast cells; biopsy 


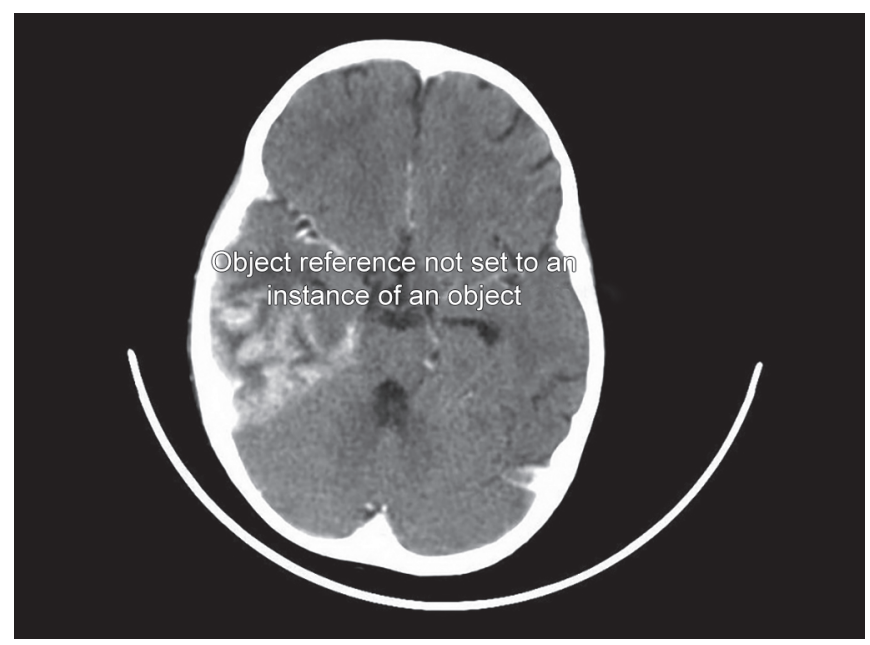

Fig. 1: CT brain showing right temporal suspected infarct, evolving abscess

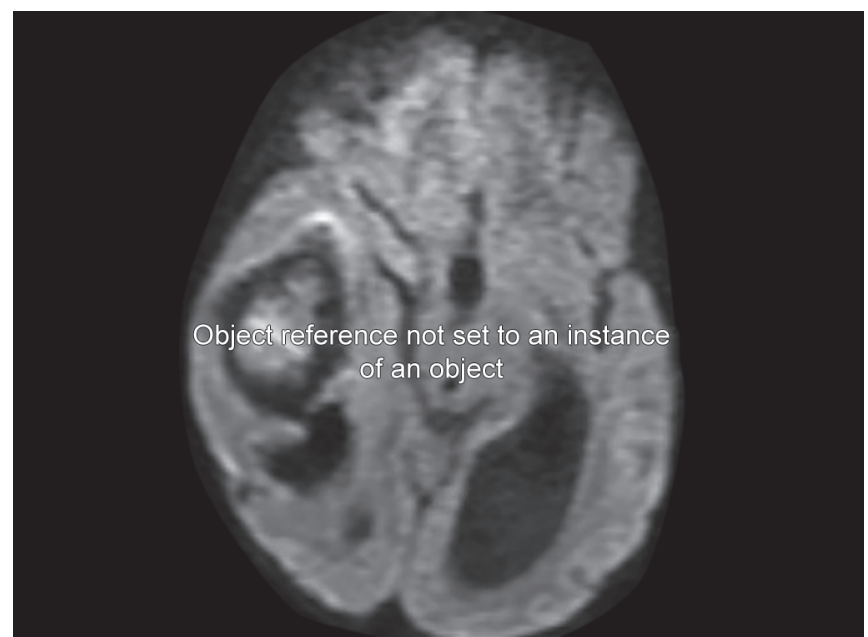

Fig. 3: MRI brain showing right temporal lobe abscess with hydrocephalus

showed significant fibrosis interspersed with marrow. However, flow cytometry ruled out the possibility of leukemia. The panel for hemophagocytic lymphohistiocytosis $(\mathrm{HLH})$ was negative. In flow cytometry for immunodeficiency, immunoglobulin levels were normal, and test for retroviral infection was negative. After 72 hours of treatment, child's fever was persistent, and Glascow coma scale (GCS) was worsening with an episode of seizure and persistent respiratory distress. Computed tomography (CT) of the brain showed right temporal lobe hypoattenuation, suggestive of acute infarct or evolving abscess with perifocal edema (Figs 1 and 2). A CT scan of the chest showed tree in bud appearance in bilateral lower lobes and consolidation of predominantly posterior lobes bilaterally. The child was investigated for TB which showed positive gastric aspirate for AFB, and catridge based nucleic acid amplification test (CB-NAAT) confirmed $M$. tuberculosis sensitive to rifampicin. Therefore, the child was started on category I antituberculous therapy (ATT). Culture also grew M. tuberculosis sensitive to first-line ATT drugs.

On day 10 of admission, because of repeated seizures and persistently depressed sensorium, magnetic resonance imaging of the brain was done, which showed cerebral abscess with obstructive hydrocephalus (Fig. 3). The abscess was drained, and Ventriculoperitoneal (VP) shunt was done by neurosurgeons.

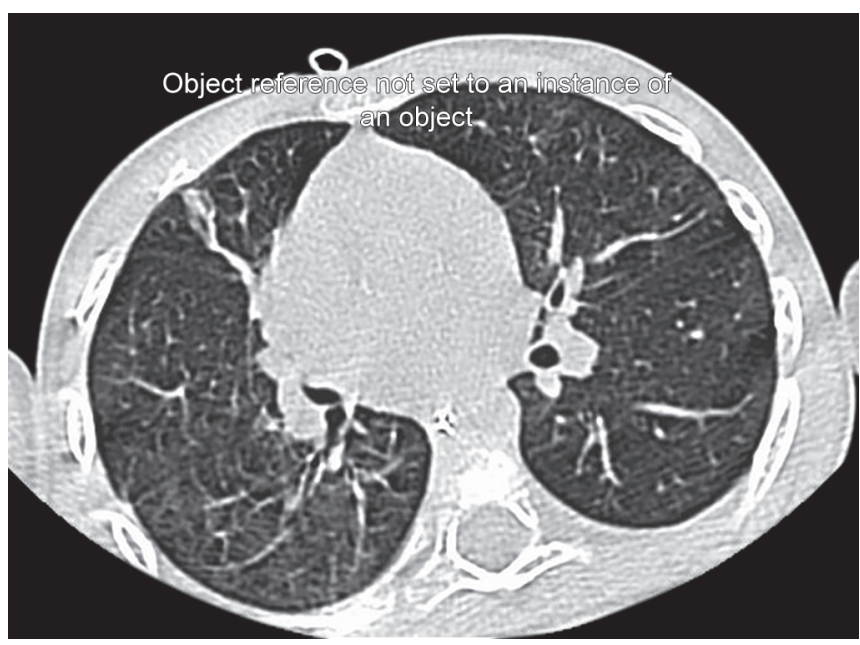

Fig. 2: CT thorax showing tree in bud appearance in lungs

The child gradually improved, was afebrile from 25th day, and was discharged on ATT. On follow-up after 4 weeks, the child had good weight gain with neurological sequelae in the form of spasticity and weakness.

\section{Discussion}

Extrapulmonary TB can present with various hematological manifestations, including pancytopenia. ${ }^{5}$ Pancytopenia can be due to hypersplenism, maturation arrest, ${ }^{1} \mathrm{HLH}^{6}{ }^{6}$ or infiltration of the bone marrow by caseating or noncaseating granulomas causing reversible or irreversible fibrosis. Singh et al. described various hematological manifestations in patients with both pulmonary and extrapulmonary TB. ${ }^{5}$ Normocytic normochromic anemia, leukopenia, neutropenia, lymphocytopenia, monocytopenia, leukocytosis, neutrophilia, lymphocytosis, monocytosis, and pancytopenia were described. Thrombocytopenia was more common in patients with disseminated/miliary TB, whereas thrombocytosis was more common in patients with pulmonary TB.

Disseminated TB remains a diagnostic challenge because the presentations are nonspecific. The index case though presented with features of TLS, but bone marrow was not suggestive of malignancy. Subsequent investigations showed a positive gastric aspirate smear for AFB, and culture for TB was positive.

Extrapulmonary TB is considered a treatable disease with a good outcome, requiring strict compliance. ${ }^{7}$ When it presents with bone marrow involvement, the outcome depends largely on timely diagnosis and early initiation of treatment. ${ }^{5}$ The index case responded clinically with the overall well-being of the child and pancytopenia resolved.

Among the nonhematopoietic diseases which cause or occur concomitantly with myelofibrosis, TB shows a frequent association, suggesting a possible relationship between the two entities. Myelofibrosis is rare in children, ${ }^{8}$ and fewer than 100 cases of pediatric myelofibrosis have been reported worldwide. In our case, bone marrow biopsy revealed significant fibrosis with preserved cellularity, but we could neither demonstrate granulomas in the bone marrow biopsy sample nor smear positivity in the aspirate. Therefore, we could not conclude direct bone marrow involvement except marked fibrosis of bone marrow, which is seen in many other causes along with TB. 
Tumor lysis syndrome is an oncological emergency, resulting from the rapid extravasation of intracellular contents into the blood due to the death of tumor cells. It is characterized by hyperkalemia, hyperphosphatemia, hyperuricemia, and hypocalcemia. In children, hematological malignancies such as acute lymphoblastic leukemia, acute myelogenous leukemia, and Burkitt lymphoma are common causes. The index case had all the parameters of TLS and the possibility of malignancy being considered. However, investigations to confirm malignancy were unyielding. In humans, uric acid is the final oxidative product of purine metabolism through the action of xanthine oxidase or xanthine dehydrogenase and is then excreted from the body through urine. In case of severe infection, ischemia and hypoxemia of many organs activate xanthine oxidase in the capillary endothelium to act upon xanthine and hypoxanthine and convert them into uric acid causing hyperuricemia like as in our child. ${ }^{9}$ Many studies reported that the serum levels of uric acid could reflect the severity and prognosis of sepsis. ${ }^{10-12}$

\section{CONCLUSION}

Tuberculosis is endemic in our country, and a high index of suspicion of disseminated TB is needed in cases of severe sepsis that do not respond to the routine treatment. Disseminated TB can very much present with features of TLS, especially in the setting of AKI, and one must be considered especially in a malnourished child.

\section{References}

1. Avasthi R, Mohanty D, Chaudhary SC, Mishra K. Disseminated tuberculosis: interesting hematological observations. J Assoc Physicians India 2010;58(4):243-244.
2. Abu-Alfa AK, Younes A. Tumor lysis syndrome and acute kidney injury: evaluation, prevention, and management. Am J Kidney Dis 2010;55(Suppl 3):S1-S13. DOI: 10.1053/j.ajkd.2009.10.056.

3. Cairo MS, Coiffier B, Reiter A, Younes A. Recommendations for the evaluation of risk and prophylaxis of tumor lysis syndrome (TLS) in adults and children with malignant diseases: an expert TLS panel consensus. Br J Haematol 2010;149(4):578-586. DOI: 10.1111/j.13652141.2010.08143.x.

4. Gertz MA. Managing tumor lysis syndrome in 2010. Leuk Lymphoma 2010;51(2):179-180. DOI: 10.3109/10428190903488788.

5. Singh KJ, Ahluwalia G, Sharma SK, Saxena R, Chaudhary VP, Anant $M$. Significance of hematological manifestations in patients with tuberculosis. J Assoc Physicians India 2001;49:788-794.

6. Le Hô H, Barbarot N, Desrues B. Pancytopenia in disseminated tuberculosis: think of macrophage activation syndrome. Rev Mal Respir 2010;27(3):257-260. DOI: 10.1016/j.rmr.2010.02.005.

7. Qasim ZA, Sarwari AR, Jilani SM. Treatment failure of tuberculosis due to concomitant pathology. J Pak Med Assoc 2003;53(8):367-369.

8. Hashim MS, Kordofani AY, el Dabi MA. Tuberculosis and myelofibrosis in children: a report. Ann Trop Paediatr 1997;17(1):61-65. DOI: 10.1080/02724936.1997.11747865.

9. Meneshian A, Bulkley GB. The physiology of endothelial xanthine oxidase: from urate catabolism to reperfusion injury to inflammatory signal transduction. Microcirculation 2002;9(3):161-175. DOI: 10.1038/ sj.mn.7800136.

10. Feig DI, Kang D-H, Johnson RJ. Uric acid and cardiovascular risk. N Engl J Med 2008;359(17):1811-1821. DOI: 10.1056/NEJMra0800885.

11. MacKinnon KL, Molnar Z, Lowe D, Watson ID, Shearer E. Measures of total free radical activity in critically ill patients. Clin Biochem 1999;32(4):263-268. DOI: 10.1016/S0009-9120(98)00109-X.

12. Tsai K, Hsu TG, Kong CW, Lin K, Lu F. Is the endogenous peroxyl-radical scavenging capacity of plasma protective in systemic inflammatory disorders in humans. Free Radic Biol Med 2000;28(6):926-933. DOI: 10.1016/S0891-5849(00)00180-5. 